\title{
A NATURAL PRODUCT DECURSIN ENHANCES THE RADIOSENSITIZATION OF IONIZING RADIATION AGAINST DMBA-INDUCED TUMOR
}

\author{
ADEEB SHEHZAD ${ }^{1 *}$, HIRA ZAHID ${ }^{1}$, WAZIR MUHAMMAD ${ }^{2}$, RIZWAN AHMAD ${ }^{3}$, EBTESAM A. AL-SUHAIMI ${ }^{4 *}$ \\ ${ }^{1}$ Department of Biomedical Engineering and Sciences, SMME, National University of Sciences and Technology, H-12, Islamabad, Pakistan. \\ ${ }^{2}$ Department of Therapeutic Radiology, Yale School of Medicine, Yale University, New Haven, CT, USA. ${ }^{3}$ Department of Biology, Natural \\ Products and Alternative Medicines, College of Clinical Pharmacy, Imam Abdulrahman Bin Faisal University, Saudi Arabia. ${ }^{4}$ Department of \\ Biology, Institute for Research and Medical Consultations, Imam Abdulrahman Bin Faisal University, Saudi Arabia. \\ Email: adeeb.shehzad@smme.nust.edu.pk
}

Received: 15 April 2018, Revised and Accepted: 25 June 2018

ABSTRACT

Objective: Radiation therapy has gained significant attention for the treatment and prevention of solid and malignant human tumors. However, after periodical exposures, radiation therapy losses its efficacy against cancer cells displaying radio-resistant phenotypes. Therefore, decursin might improve the efficiency of radiotherapy against a variety of human cancers.

Methods: The chemopreventive efficacy of decursin was evaluated against B16F10 cancer cell lines and DMBA/croton oil-induced skin carcinogenesis in $B A L B / c$ mice. Decursin was administered intraperitoneal at the dose of $20 \mathrm{mg} / \mathrm{kg} /$ day for 8 weeks following exposure to 5 Gy of ionizing radiation (IR) after 1 month of DMBA application. Western blot was performed for underlying mechanism of radioresistance.

Results: Decursin suppressed the proliferation and viability of melanoma cancer cell lines in a concentration- and time-dependent manner. The in vivo data collected from mice model revealed that decursin reduced the precancerous skin lesions and the incidence of tumor bearing in radiation-exposed

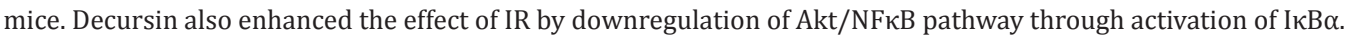

Conclusion: Our results suggest that the activation of Akt/NFkB establishes a pro-survival response to radiation that may account for the development of radioresistance. Decursin blocks the abnormal expression of these proteins and potentiates the radiotherapeutic effect.

Keywords: Decursin, Ionizing radiation, DMBA, Inflammation, Squamous carcinoma.

(C) 2019 The Authors. Published by Innovare Academic Sciences PvtLtd. This is an open access article under the CC BY license (http://creativecommons. org/licenses/by/4. 0/) DOI: http://dx.doi.org/10.22159/ijap.2019.v11s3.M1001

\section{INTRODUCTION}

Ionizing radiation (IR) is an established beneficial treatment modality for the treatment of pre- and post-operative cancer, which selectively target and destroy cancer cells [1]. Radioresistance is a biological phenomenon which limits and decreases the maximal therapeutic efficacy of radiotherapy for the treatment of tumors [2]. Among various human cancers, melanoma has been believed to be a radio-resistant tumor type as compared to other types of cancers [3]. Studies have reported various plant isolated compounds for radiosensitization as well as radioprotection [4]. Therefore, low cost and less toxic radiosensitizers are urgently required, which can reduce the radiation dose-response threshold for cancer cells and provide radioprotection to normal cells [4].

PI3k/Akt pathway has been involved in tumor cell proliferation and intrinsic radioresistance [5]. In melanoma, aberrant activity of the PI3k/ Akt pathway has been demonstrated to promote melanogenesis [5]. A number of studies have shown that specific inhibition of PI3k/ Akt/mTOR pathway has enhanced the radiosensitivity of various cancer cell lines both in vitro and in vivo [6]. On activation, PI3k/Akt pathway also regulates the expression of downstream targets such as $\mathrm{NF} \kappa \mathrm{B}$, which further potentiates the process of radioresistance. $\mathrm{NF \kappa B}$ activation constitutively leads to radioresistance. Inhibition of NFKB activation can lead to an increase in the efficacy of radiation therapy [7]. Therefore, downregulation of $\mathrm{NF \kappa B}$ is a promising target to enhance radiosensitivity [7].

Decursin is a naturally occurring coumarin compound isolated from the roots of Korean Angelica also known as Angelica gigas Nakai [8].
Decursin exerts its anticancer effects by inducing apoptosis through activation of caspase 8,9 , and 3 , downregulation of antiapoptotic proteins such as Bcl-2 and Bcl-xL, by inhibiting vascular endothelial growth factor (VEGF)-induced angiogenesis, by inducing cell cycle arrest through the downregulation of cyclin D1, and by modulating various signaling pathways including NFKB and PI3K pathways $[9,10]$.

Previously, we have summarized and reported the anti-inflammatory and synergistic role of decursin in various cancers [11]. In the current study, we have investigated the role of decursin in modulating the radioresponse of melanoma cancer in vitro and in vivo. Decursin enhanced radiosensitization through downregulation of $\mathrm{NFKB}$ and PI3K.

\section{METHODS}

\section{Cell culture and treatment}

B16F10 cells of Mus musculus skin melanoma were obtained from ATCC, CRL- $6475^{\mathrm{TM}}$. Cells were cultured at a density of $5 \times 10^{5}$ in RPMI1640 medium containing $10 \%$ heat-inactivated fetal bovine serum, L-glutamine and supplemented with $1 \%(\mathrm{v} / \mathrm{v})$ antibiotic-penicillin streptomycin (Gibco, Invitrogen Corporation) at $37^{\circ} \mathrm{C}$ in a $5 \% \mathrm{CO}_{2}$ humidified atmosphere. $70 \%$ confluent growing cells were seeded at $1 \times 10^{6}$ cells per dish followed by exposure to various concentrations $(2,5,10,20,40,80$, and $100 \mu M)$ of decursin $(\geq 97 \%$ Sigma-Aldrich, USA). For 3-[4,5-dimethylthiazol-2-yl]-2,5-diphenyltetrazolium bromide (MTT) assay, cells were exposed to various concentrations of decursin for next $24 \mathrm{~h}$. After this, medium was removed and $50 \mu \mathrm{l}$ of MTT solution ( $5 \mathrm{mg} / \mathrm{mL}$ in phosphate-buffered saline) was added to each well. After $3 \mathrm{~h}$, the MTT solution was removed and $200 \mu \mathrm{l}$ of 


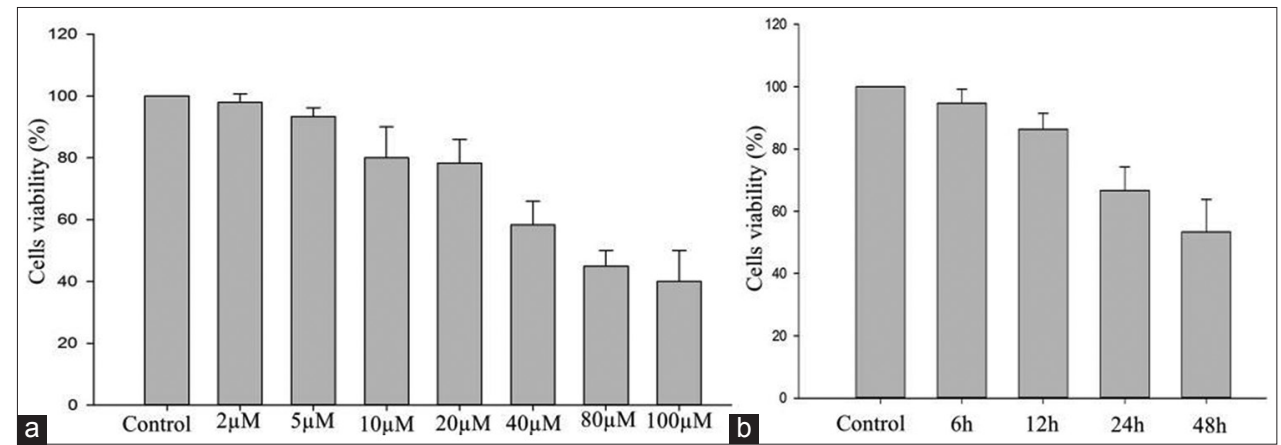

Fig. 1: (a) Concentration0dependent effect of decursin against B16F10 cell lines. (b) Time-dependent effect of $40 \mu \mathrm{L}$ of decursin against B16F10 cell lines. p $\leq 0.05$

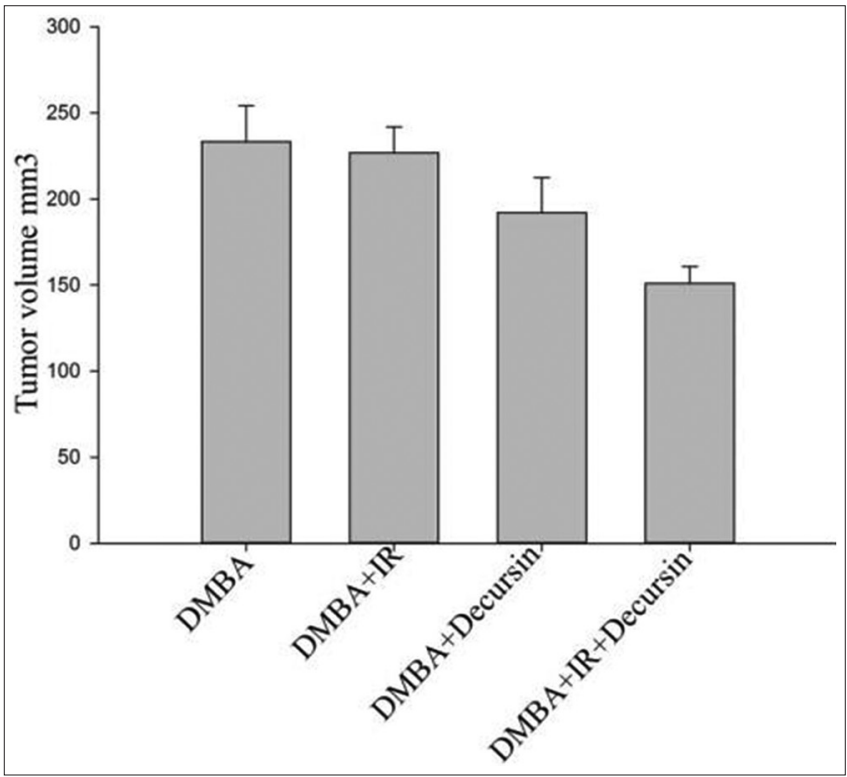

Fig. 2: Decursin sensitizes DMBA-induced tumors to radiation in BALB/C mice. Decursin and IR together reduced tumor size as compared to their individual treatments. Tumor volumes were measured using Vernier calipers, calculated as described in materials and methods. $p \leq 0.05(n=6)$

dimethyl sulfoxide was added and analyzed by microplate reader (BioTek, USA) at $570 \mathrm{~nm}$ in a triplicate.

\section{Experimental design}

BALB/C male mice (27-30 g, 5-6 weeks) were purchased from the National Institute of Health (NIH, Islamabad, and were divided into four groups, each containing six mice. Our experimental protocol was approved by the Institutional Animal Care and Ethics Committee, in line with international laws and policies (NIH Guide for the Care and Use of Laboratory Animals, NIH publication No. 85-23, 1985). These groups were assigned to different treatments as follows: Group A (DMBA/ Croton oil): The animals in this group received topical applications of DMBA in acetone $(100 \mu \mathrm{L})$ at a dose of $400 \mathrm{nmol}$ per application at an interval of $72 \mathrm{~h}$ per mouse. Starting from the 8 days after the first DMBA application, croton oil $(1 \% \mathrm{w} / \mathrm{v})$ in acetone $(100 \mu \mathrm{L})$ was applied twice in a week for a total of 8 weeks. Group B mice were exposed to $5 \mathrm{~Gy} /$ month of IR after induction of tumor with DMBA/croton oil. Group C (decursin $20 \mathrm{mg} / \mathrm{kg}$ ip injection): The animals in this group were applied with DMBA/croton oil following concomitant treatment with ip injection of decursin. Group D received decursin and IR after treatment with DMBA/croton oil. During the 8 weeks of experimental period, the animals were observed daily for the appearance of skin papillomas and the tumor volume was recorded. At the start of 9 weeks, all the mice were sacrificed and the skin samples were collected.

\section{Western blotting}

In brief, proteins were extracted from the developed tumor of different treatment groups through tissue lysis buffer. Proteins were transferred onto nitrocellulose membranes $(0.2 \mu \mathrm{M}$, Schleicher and Schuell). Membranes were blocked using 5\% non-fat dry milk and probed with primary antibody in TBS containing 3\% non-fat dry milk and $0.1 \%$ Tween 20. Antibodies in the ratio of 1:1000 v/v were used and detected using an enhanced chemiluminescence kit (Amersham Corp).

\section{RESULTS}

Decursin inhibited B16F10 cells proliferation

Cell viability assay determines the inhibitory concentration of the drug, which has therapeutic effect. B16F10 cell lines were treated with various concentrations $(0-100 \mu \mathrm{M})$ of decursin. As shown in Fig. 1a, decursin reduced the cells viability of B16F10 cells in a concentration-dependent manner. Furthermore, we checked the time-dependent effects of $40 \mu \mathrm{M}$ of decursin various time periods. Decursin significantly reduced the cells viability after $12 \mathrm{~h}$ (Fig. 1b). These results demonstrate the therapeutic potential of decursin in vitro against melanoma cell lines.

\section{Decursin reduced DMBA-induced tumor in BALB/C}

We evaluated the combined effects of decursin and IR against the growth of DMBA-induced tumor model. As shown in Fig. 2, combined administration of decursin and IR reduced the tumor growth as compared to their individual effect. Tumor volume was measured according to the formula: $V=4 / 3 \pi W^{2} L$ (short size ${ }^{2} \times$ long size $/ 2\left(\mathrm{~mm}^{3}\right)$. The mice were sacrificed for the isolation of tumor after the $8^{\text {th }}$ week.

Decursin inhibited the DMBA-induced expression of NFKB and Akt In our study, DMBA treatment activates Akt and NFkB, whereas degrad IkB (Fig. 3). However, decursin treatment reversed the carcinogenic and tumorigenic effect of DMBA. Decursin potentiated the effects of IR in DMBA-induced tumor (Fig. 3). One of the known activators of IKK signaling pathway is Akt, which also mediates radioresistance through induction of pro-survival signaling. Decursin significantly inhibited DMBA-induced Akt and NFkB (Fig. 3).

\section{DISCUSSION}

Skin cancers are counted among curable cancers, but the development of resistant phenotype makes it hard to treat with standard chemo and radiotherapy $[12,13]$. The study was conducted to investigate the radiosensitizing effect decursin in DMBA-induced carcinoma. For this purpose, B16F10 cells and carcinogen model were exposed to decursin and IR. Decursin reduced the cells viability in a concentration- and timedependent manner. Decursin reduced the cancer lesions in squamous cell carcinoma and potentiated the effect of IR in DMBA-induced tumor model. The previous studies have shown that activation of Akt and $\mathrm{NF \kappa B}$ provides a pro-survival response to radiation that may account for the development of radioresistance [14,15]. Decursin activates $\mathrm{IKB} \alpha$ and inhibits Akt/NFKB pathway and boosts the efficacy of radiotherapy. 


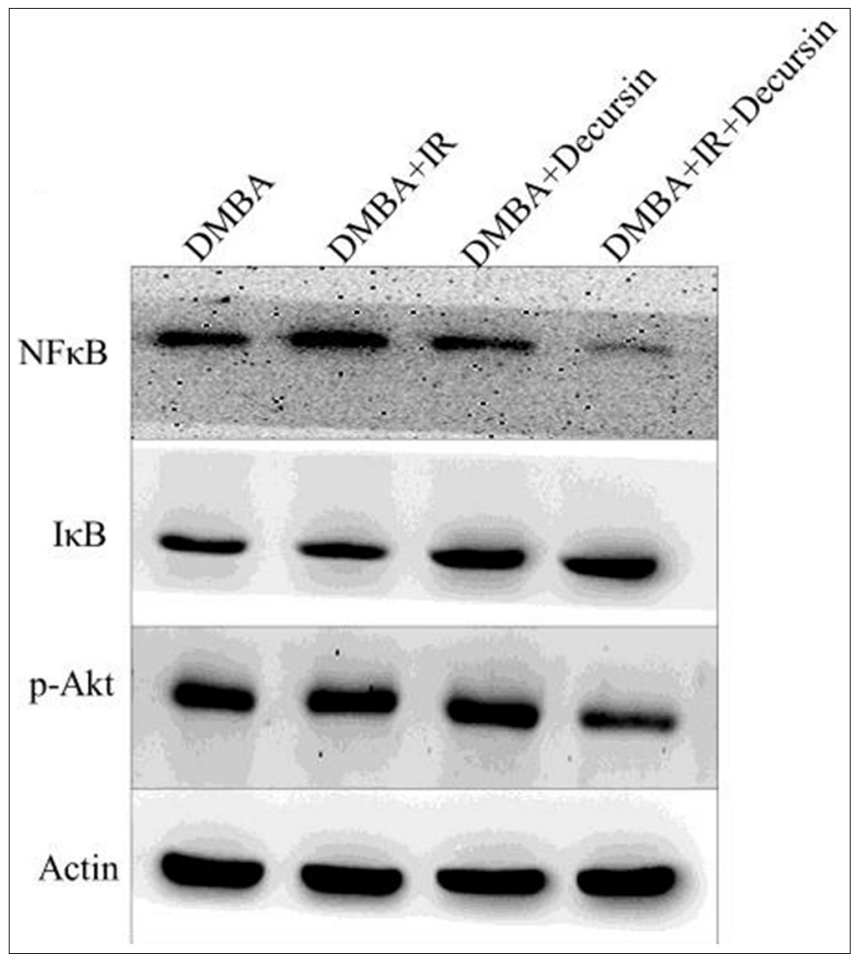

Fig. 3: Decursin suppressed the expression of NFKB and Akt in DMBA-induced tumor mice. Developed tumors were isolated,

homogenized and total protein was isolated from cells and analyzed by western blotting. Actin was used as a loading control

There is accumulating evidence that PI3k/Akt and NFKB signaling pathways are involved in radioresistance [12,14]. Activation of Akt causes downregulation of tumor suppressor gene phosphatase and tensin homolog and inhibits the process of apoptosis [16,17]. Furthermore, Akt has been reported to phosphorylate the NFKB, increasing the transcription of NFKB downstream targets involved in cancer development and therapeutic resistance $[18,19]$. Therefore, inhibition of Akt will suppress the activation of $\mathrm{NFKB}$ in malignant melanoma cells [18]. Decursin treatment decreased the expression of p52 subunit of NFkB in a DMBA-treated group. The expression of IкB was also increased in the decursin-treated group. Our results indicate that decursin has the ability to block Akt and NFKB activation in DMBAinduced tumor model. Decursin can provide alternate strategy to potentiate the therapeutic effect of IR.

The underlying mechanism of tumor resistance is mediated through multiple interrelated signal transduction pathways. Deep understanding of these multiple molecular processes underlying resistance to cancer therapeutics has led to the demand for the less toxic and cost-effective novel agents $[20,21]$.

\section{CONCLUSION}

Decursin has the ability to reduce the cells viability of B16F10 cells in a concentration- and time-dependent manner. Decursin also enhances the effect of IR by downregulating the expression of Akt/NFkB, which is involved in cancer cells survival and radioresistance. Therefore, decursin-induced radiosensitization may have potential therapeutic effect in cancer radiotherapy.

Further in vitro and in vivo studies are needed to investigate the molecular mechanism of decursin in various cancers to further advocate the radiosensitizer role. Furthermore, pharmacodynamics, pharmacokinetics, and safety studies of decursin are further needed to confirm the detail mechanism of action with optimum dose and dosage form in cancer chemotherapy.

\section{CONFLICTS OF INTEREST STATEMENT}

All authors have declared no conflicts of interest.

\section{REFERENCES}

1. Qureshi M, Al-Suhaimi EA, Wahid F, Shehzad O, Shehzad A. Therapeutic potential of curcumin for multiple sclerosis. Neurol Sci 2018:39:207-14.

2. Shehzad A, Park JW, Lee J, Lee YS. Curcumin induces radiosensitivity of in vitro and in vivo cancer models by modulating pre-mRNA processing factor 4 (Prp4). Chem Biol Interact 2013;206:394-402.

3. Chang DT, Amdur RJ, Morris CG, Mendenhall WM. Adjuvant radiotherapy for cutaneous melanoma: Comparing hypofractionation to conventional fractionation. Int J Radiat Oncol Biol Phys 2006;66:1051-5.

4. Camphausen K, Tofilon PJ. Combining radiation and molecular targeting in cancer therapy. Cancer Biol Ther 2004:3:247-50.

5. Al-Suhaimi EA. Curcumin induces apoptosis of 3T3-L1 adipocytes and affects molecular signals of adiponectin, AMPK and PKA. An Real Acad Farm 2104;80:720-34

6. Chang L, Graham PH, Hao J, Ni J, Bucci J, Cozzi PJ, et al. Acquisition of epithelial-mesenchymal transition and cancer stem cell phenotypes is associated with activation of the PI3K/Akt/mTOR pathway in prostate cancer radioresistance. Cell Death Dis 2013;4:e875.

7. Nakanishi C, Toi M. Nuclear factor-kappaB inhibitors as sensitizers to anticancer drugs. Nat Rev Cancer 2005;5:297-309.

8. Kim BS, Seo H, Kim HJ, Bae SM, Son HN, Lee YJ, et al. Decursin from Angelica gigas nakai inhibits B16F10 melanoma growth through induction of apoptosis. J Med Food 2015;18:1121-7.

9. Kim JH, Jeong JH, Jeon ST, Kim H, Ock J, Suk K, et al. Decursin inhibits induction of inflammatory mediators by blocking nuclear factorkappaB activation in macrophages. Mol Pharmacol 2006;69:1783-90.

10. Lee SH, Lee JH, Kim EJ, Kim WJ, Suk K, Kim JH, et al. A novel derivative of decursin, CSL-32, blocks migration and production of inflammatory mediators and modulates PI $3 \mathrm{~K}$ and NF- $\mathrm{KB}$ activities in HT1080 cells. Cell Biol Int 2012;36:683-8.

11. Shehzad A, Parveen S, Qureshi M, Subhan F, Lee YS. Decursin and decursinol angelate: Molecular mechanism and therapeutic potential in inflammatory diseases. Inflamm Res 2018;67:209-18.

12. Munshi A, Kurland JF, Nishikawa T, Chiao PJ, Andreeff M, Meyn RE, et al. Inhibition of constitutively activated nuclear factor-kappaB radiosensitizes human melanoma cells. Mol Cancer Ther 2004;3:985-92.

13. Al-Suhaimi E. Molecular mechanisms of leptin and pro-apoptotic signals induced by menadione in hepG2 cells. Saudi J Biol Sci 2014;21:582-8.

14. Schön M, Wienrich BG, Kneitz S, Sennefelder H, Amschler K, Vöhringer $\mathrm{V}$, et al. KINK-1, a novel small-molecule inhibitor of IKKbeta, and the susceptibility of melanoma cells to antitumoral treatment. J Natl Cancer Inst 2008;100:862-75.

15. Yu CC, Hung SK, Lin HY, Chiou WY, Lee MS, Liao HF, et al. Targeting the PI3K/AKT/mTOR signaling pathway as an effectively radiosensitizing strategy for treating human oral squamous cell carcinoma in vitro and in vivo. Oncotarget 2017;8:68641-53.

16. Al Suhaimi EA. Effect of Corriandrum sativum, a common herbal medicine on endocrine and reproductive organ structure and function. Internet J Altern Med 2008;7:1540-2584.

17. Stahl JM, Sharma A, Cheung M, Zimmerman M, Cheng JQ, Bosenberg MW, et al. Deregulated akt3 activity promotes development of malignant melanoma. Cancer Res 2004;64:7002-10.

18. AL-Suhaimi EA, Fadwa M, Ravinayagam V, Mohammad H. Serum adipocytokines, metabolic and immunological correlations in Type 1 diabetes mellitus (T1DM) children. Open Endocrinol J 2012;6:110-6.

19. Dhawan P, Singh AB, Ellis DL, Richmond A. Constitutive activation of akt/protein kinase $B$ in melanoma leads to up-regulation of nuclear factor-kappaB and tumor progression. Cancer Res 2002;62:7335-42.

20. Al-Suhaimi EA, Akbar A. Relationship of leptin hormone with insulin and glucose in Arabian camel (Camelus dromedarius). J Camel Pract Res 2009;16:201-7.

21. Al-Suwaigh EA, Al-Suhaimi EA. Comparative study on some biochemical constituents of plasma in male camels and goats. J Camel Pract Res 2014;12:141-3. 